\title{
Comparison of COX-2 Selective and Traditional NSAIDs on Experimental Gastric Ulcer Healing in Humans
}

\author{
Tau JA, Qureshi W, El-Zimaity HMT, Opekun AR \\ and Graham DY* \\ Department of Medicine, Veterans Affairs Medical Center \\ and Baylor College of Medicine, Houston, TX, USA \\ *Corresponding author: David Y Graham, Michael E. \\ DeBakey Veterans Affairs Medical Center, RM 3A-390A \\ (111D), 2002 Holcombe Boulevard, Houston, TX 77030, \\ USA
}

Received: June 22, 2021; Accepted: July 14, 2021;

Published: July 21, 2021

\begin{abstract}
Background: Nonsteroidal anti-inflammatory drugs impair gastrointestinal ulcers healing. This study evaluated the role of cyclooxygenase isozymes COX1 and COX-2 in the healing of acute gastric ulcers in humans.

Methods: This was an open-label, endoscopist-blind, parallel-group study, age and sex matched at baseline in normal volunteers. At endoscopy, we took four large jumbo forceps gastric mucosal biopsies (2 from each of the antrum and corpus). Subjects received celecoxib $200 \mathrm{mg}$ bid), naproxen $500 \mathrm{mg}$ bid), nabumetone $1000 \mathrm{mg}$ bid or placebo until end of study. Endoscopies were performed after 5 days and every 3 days until complete re-epithelialization of all lesions or 30 days. Survival analysis was used to compare time-to-healing defined as the day with complete re-epithelialization of all ulcers.

Results: Fifty-two subjects completed the study, each received four biopsyinduced gastric ulcers (204 total ulcers; the majority included the muscularis mucosa). The mean time-to-healing was $9.4 \pm 0.4$ days with placebo, $10.5 \pm 0.4$ with celecoxib, $11.1 \pm 0.6$ with naproxen, and $12.3 \pm 0.9$ with nabumetone. The time to healing of each ulcer or all ulcers was significantly delayed compared to placebo with naproxen $(p=0.01)$ and nabumetone $(p=0.002)$ but not with celecoxib $(p=0.07)$.
\end{abstract}

Conclusion: The COX-1 preferential inhibitor naproxen and the balanced COX-1/COX-2 inhibitor nabumetone significantly delayed the healing of ulcers. With the COX-2 specific inhibitor celecoxib, healing was delayed but not significantly. Synthesis of COX-1 derived prostaglandins appears to be important in the healing of gastric ulcers in humans.

Keywords: Helicobacter pylori; Nonsteroidal anti-inflammatory drugs; Celecoxib; Nabumetone; Naproxen; Placebo; Human volunteers; Experimental ulcer; Ulcer healing

\section{Introduction}

Non-Steroidal Anti-Inflammatory Drugs (NSAIDs) are widely available over the counter and by prescription and are widely used for treatment of pain and inflammation [1-4]. NSAID use is however not without risk [5]. The risk of developing a serious, life-threatening event attributable to NSAID use increases with age, concurrent corticosteroid use, concomitant Helicobacter pylori infection, antiinflammatory dose, past history of an ulcer or ulcer complication, as well as the use of multiple NSAIDs including aspirin $[1,6,7]$. The major gastrointestinal adverse event associated with NSAID use is gastroduodenal ulceration, which may result in gastrointestinal bleeding, perforation or obstruction [8]. As the prevalence of Helicobacter pylori infection has declined, Non-Steroidal Anti-Inflammatory Drug (NSAID) has become a major cause of gastro-duodenal ulcers [9].

The primary event leading to mucosal damage is related to the ability of NSAIDs to inhibit mucosal Cyclooxygenase (COX), which exist in two forms (COX-1 and COX-2). COX-1 is constitutively expressed and is primarily responsible for maintenance of both gastric mucosal integrity and mucosal repair [10]. COX-2 is predominantly responsible for inflammation. It was initially believed that NSAIDs with predominately COX-2 inhibition would allow targeted therapy and allow clinicians to relief pain and inflammation without inhibiting normal COX-1 related mucosal defense. Thus, pain relief would be obtained with a reduction in NSAID-associated risks of significant gastrointestinal adverse events. Several selective COX-2 inhibitors were introduced and compared with COX-1 inhibitors concerning their propensity to cause mucosal damage and more importantly, clinically significant outcomes of perforation, ulceration, or major bleeding (PUBs) [11]. Despite the admonition that one should use NSAIDs at their lowest effective dose or the shortest time, the study paradigm typically consisted of comparisons with maximum allowable doses of traditional NSAIDs. This proved difficult in part because of the rapid decline of Helicobacter pylori infections and the fact that concomitant aspirin appeared to abrogate any potential benefit of specific COX-2 inhibitors. Because of the discovery of significant cardiovascular side effects all selective COX-2 inhibitors except celecoxib were withdrawn and the dosage of celecoxib was restricted $[12,13]$. A subsequent large-scale study that compared celecoxib to naproxen and ibuprofen taken with esomeprazole confirmed the low cardiovascular risk with celecoxib. In this treatment paradigm, major gastrointestinal events although uncommon were significantly less frequent with celecoxib than high dose naproxen or ibuprofen $[14,15]$. 
Traditional NSAIDs differ in their analgesic properties in relation to their inflammatory activity, which also allows clinicians to select NSAIDs based on their goal (e.g., analgesia, inflammatory activity, or both) [16]. For example, 200mg of ibuprofen has primarily analgesic activity with minimal anti-inflammatory activity. In contrast, piroxicam has analgesic activity only at a full anti-inflammatory activity. Nabumetone is a nonacidic prodrug with a metabolite (6 MNA) that inhibits both COX 1 and COX 2 in vivo [17]. Nabumetone is also considered to be "preferential" for COX 2 with only modest COX-1 activity and along with Etodolac, have been proposed to possibly have increased safety when the indication is for analgesia and high anti-inflammatory activity is not required $[16,18]$.

Both traditional and COX-2 inhibitors have also been shown to delay the healing of experimental ulcers in animal models. Both can also cause acute gastric mucosal damage, including ulcers $[19,20]$. This study was designed to compare the effects of traditional NSAIDs and selective COX-2 inhibitors on the healing of acute experimental gastric ulcers in humans. The hypothesis was that COX-2 inhibitors would not decrease the rate of mucosal lesion healing compared to placebo and would produce similar delayed healing rates similar to that of the traditional NSAIDs, naproxen, or nabumetone.

\section{Methods}

This study was an open label, placebo-controlled endoscopist blind, and parallel-group study to compare the effects of celecoxib, nabumetone, and naproxen on the healing of iatrogenic induced gastric ulcers in human volunteers (IND 58,489). It was based on the premise that routine mucosal biopsy with jumbo forceps causes small acute ulcers that heal rapidly among subjects receiving placebo. The NSAIDs chosen for the three test groups were celecoxib, nabumetone, and naproxen (a potent COX-1-preferential NSAID) or placebo. All procedures performed in studies involving human participants were in accordance with the ethical standards of the institutional and/or national research committee and with the 1964 Helsinki Declaration and its later amendments or comparable ethical standards. The study was approved by the Baylor Affiliates Review Board for Human Studies and the Houston Veteran's Affairs Medical Center Human Research Committee.

After obtaining informed consent, subjects were enrolled if they were aged 18 to 55 years, found to be in good health by medical history, physical examination and routine laboratory tests, understood the stringent requirements of the study protocol Demographic information was recorded for each subject, including gender, occupation, and ethnicity.

Subjects were excluded from the study if they had any significant medical conditions including peptic ulcer disease, gastrointestinal hemorrhage, cardiovascular disease, renal disease, hepatic disease, pulmonary disease, uncontrolled diabetes, hypercalcemia, blood dyscrasia, coagulopathy, required anticoagulation therapy or active neoplastic disease. Other exclusion criteria included a history of aspirin intolerance; aspirin-induced bronchospasm, asthma, nasal polyps, angioedema, alcohol or drug abuse within 5 years of screening or pregnancy as determined by serum HCG titer. Subjects were also excluded if they had admitted to using antacids, protonpump inhibitors, or $\mathrm{H} 2$-antagonists for symptoms of dyspepsia more than twice during the month preceding enrollment. Other exclusionary drug use included warfarin, corticosteroids (parenteral or by mouth), NSAIDs (including over-the-counter products), cold and sinus remedies that contained NSAIDs, COX-2 inhibitors, or any other medication with known potential for inducing gastrointestinal mucosal injury.

Once subjects were generally qualified to participate, they underwent upper gastrointestinal endoscopy using a Pentax upper gastrointestinal endoscope. Except for topical oro-pharyngeal anesthesia, they were not sedated. If the gastric mucosa appeared normal by gross visual surveillance and the subjects tolerated the procedure easily, they were cleared for further participation with iatrogenic ulcer induction and medication randomization. Preexisting gastric mucosal lesions resulted in disqualification.

Qualified subjects received 4 jumbo (3.3mm, MicroVasive Radial Jaw Single-use Biopsy Forceps, Boston Scientific, Watertown MA) gastric mucosal biopsies that included the muscularis mucosa; 2 from the gastric antrum and two from the gastric corpus or 4 per subject. Each biopsy was oval with fixed dimensions approximating $2.8 \times 5.8$ $\mathrm{mm}$. Biopsies fixed in $10 \%$ buffered formalin and were processed using standard histopathological methods including hematoxylin and eosin staining methods and by the El-Zimaity staining method for evaluation of residual inflammation [21]. Histopathological observations were recorded including the presence or absence of $H$. pylori infection, and Polymorphonuclear Cells (PMN's). Subject were enrolled prior to availability of the histology results.

Following endoscopy, patients were randomized and received either celecoxib (200mg bid), naproxen (500mg bid), nabumetone $(1,000 \mathrm{mg}$ bid) or placebo. Follow up endoscopies were performed after 5 days of drug and then at 3 day intervals until complete re epithelialization (primary outcome variable) of all lesions or a total of four weeks passed (days $0,6,9,12,15,18,21,24,27$, and 30). The NSAID was continued throughout this period. At each endoscopy, the site of each lesion was identified and an endoscopic assessment of re epithelialization was determined and recorded.

Although the study was not a completely blinded study, it was randomized, and the endoscopist was blinded to the treatment regimen received by individual subjects. All study medication was dispensed through the hospital pharmacy. Celecoxib and naproxen were commercial products. SmithKline Beecham Pharmaceuticals provided Nabumetone and matching placebo. Both the nabumetone and placebo were supplied as $500 \mathrm{mg}$ in the form of unmarked white tablets.

\section{Data analysis}

We expected the ulcers to completely re-epithelialized within 8 days with placebo and that NSAIDs use would prolong the time to healing. The analyses included the proportion with unhealed biopsy induced lesions at each time point as well at the time to healing for the group. This was evaluated two ways as the time to complete healing and as the time when one-half ( 2 or the 4 ) biopsy sites were healed. The primary analysis was placebo $v s$. naproxen and was designed to confirm the animal studies. We chose the sample size based on the premise that healing will be complete in at least $80 \%$ of those receiving placebo at day $8 v$ s. $20 \%$ of those receiving NSAIDs. The sample size of 


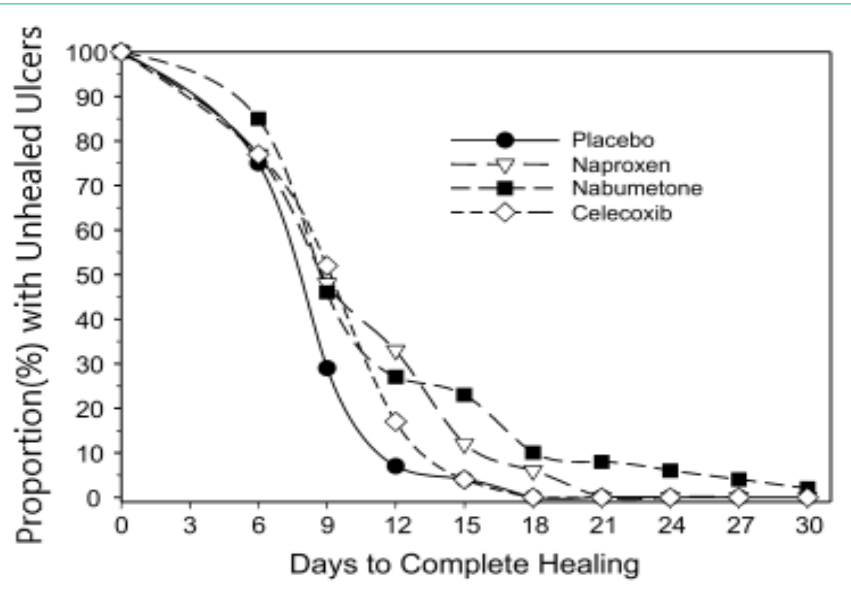

Figure 1: Days to complete healing of the acute gastric ulcers for the 4 treatment groups (Kaplan-Meier life table analysis).

13 per group for the primary analysis was calculated using SigmaStat with a power of 0.8 and alpha of 0.05 and Yates correction factor. The secondary analyses are exploratory and address the question of whether NSAIDs with lesser ability to damage the gastric mucosa react more like naproxen or like a placebo. These analyses included the proportion of patients with unhealed biopsy induced lesions and the number of lesions remaining unhealed at each time point as well at the time to healing for the group. We calculated the group means at each time point using the number of lesions unhealed for each patient. We used cumulative life table analyses to compare grouphealing rates, with particular attention to the time to $50 \%$ healing.

\section{Statistical analyses of healing of antral, corpus and all lesions}

Data were recorded using standardized case report forms and transcribe to computerized spreadsheets (Excel, Microsoft, Redmond WA). Statistical Analyses, including summary and descriptive statistics, were performed using SPSS Version 10 (2001), SPSS Inc., Chicago, IL. To determine the reproducibility of the healing lesions, the mean difference in time to healing (days) between the first and second biopsy was assessed for all study groups combined. The mean difference to healing between two biopsies, by region, was $2.6 \pm 3.1$ days for antral lesions and $1.6 \pm 2.4$ days for corpus lesions.

Life tables were constructed using six 5-day intervals from zero to 30 days to determine healing time (maximum value). During life table analyses, the Wilcoxon (Gehan) test statistic was calculated for making pairwise comparisons of healing between all possible pairs of treatment groups. Kaplan-Meier analysis was also performed using the log-rank test for pairwise comparisons. Finally, we performed Cox proportional hazards regression for comparing relative risks of healing for the various treatment groups when compared with placebo.

\section{Results}

Subjects: Fifty-two subjects, 31 men, 21 women, mean aged $35.1 \pm 10$ years, underwent an upper gastrointestinal endoscopy, and completed the trial. We screened seven additional subjects but excluded them from the study because of pre-existing gastric mucosal lesions or inability to tolerate upper gastrointestinal endoscopy without sedation.

The treatment groups in the study population were well matched for the demographic factors of age, race, and H. pylori status at baseline (Table 1). There were significantly more females in the celecoxib treatment group.

Average and median healing times for the placebo group were $11.6 \pm 0.8$ (95\% CI, 10.0-13.1) and $12.0 \pm 0.92$ (95\% CI, 10.2-13.8). The shortest median healing time observed was with Nabumetone, which was $12.0 \pm 3.4$ days (95\% CI, 5.21-18.79). Both naproxen and celecoxib had similar median healing times of 15 days, with similar standard errors near unity. The Wilcoxon test for similar healing times indicated that only the placebo-celecoxib pairwise comparison was significant $(\mathrm{p}<0.05)$. In contrast, the log -rank test for both the placebo-naproxen and placebo-nabumetone pairwise comparisons were significant. Alternatively, the Cox proportional hazards regression indicated that only nabumetone was significant.

The Wilcoxon test was likely significant for the placebo-celecoxib pairwise test because the celecoxib treatment group had an average healing time (13.6) that was lower than the median of 15 days. For skewed distributions with medians at the peak, the average tends to fall on the side with the longest tail. Thus, for the celecoxib treatment effect, the tail of the healing time distribution was skewed left with more individuals having shorter healing times. Since the Wilcoxon test is sensitive to "early dropouts" or in this study, those that healed early, the test statistic was highly significant for the celecoxib treatment. For naproxen and nabumetone, average healing times were higher than the median healing times, meaning that the healing time distribution

Table 1: Demographics of the study population.

\begin{tabular}{|c|c|c|c|c|c|}
\hline & Celecoxib $(n=13)$ & Nabumetone $(n=12)$ & Naproxen $(n=13)$ & Placebo (n=14) & Total $(n=52)$ \\
\hline Asian & 0/13 (0\%) & $1 / 12(8 \%)$ & 0/13 (0\%) & $0 / 14(0 \%)$ & $1 / 52(2 \%)$ \\
\hline Black & 4/13 (31\%) & $4 / 12(33 \%)$ & $2 / 13(15 \%)$ & $5 / 14(36 \%)$ & $15 / 52(29 \%)$ \\
\hline Hispanic & $1 / 13(8 \%)$ & $0 / 12(0 \%)$ & 0/13 (0\%) & $3 / 14(21 \%)$ & $4 / 52(8 \%)$ \\
\hline White & $8 / 13(62 \%)$ & $6 / 12(50 \%)$ & 10/13 (77\%) & $4 / 14(29 \%)$ & $28 / 52(54 \%)$ \\
\hline Other & $0 / 13(0 \%)$ & $1 / 12(8 \%)$ & 1/13 (8\%) & $2 / 14(14 \%)$ & $4 / 52(8 \%)$ \\
\hline Male & $3 / 13(23 \%)$ & $9 / 12(75 \%)$ & $9 / 13(69 \%)$ & $10 / 14(71 \%)$ & $31 / 52(60 \%)$ \\
\hline Female & 10/13 (77\%) & $3 / 12(25 \%)$ & $4 / 12(31 \%)$ & $4 / 14(29 \%)$ & $21 / 52(40 \%)$ \\
\hline H. pylori infected & $5 / 13(38 \%)$ & $5 / 12(42 \%)$ & 4/13 (31\%) & $5 / 14(36 \%)$ & 19/52 (37\%) \\
\hline Mean Age \pm SD & $34.4 \pm 12$ & $34.3 \pm 10$ & $36.6 \pm 10$ & $35.1 \pm 9$ & $35.1 \pm 10$ \\
\hline
\end{tabular}


Table 2: Sample sizes and median healing times.

\begin{tabular}{|c|c|c|c|}
\hline Treatment & $\mathbf{N}$ & Mean \pm SE (95\% Cl) & Median \pm SE (95\% Cl) \\
\hline Placebo & 14 & $11.6 \pm 0.8(10.0-13.1)$ & $12.0 \pm 0.92(10.19-13.81)$ \\
\hline Naproxen & 13 & $15.2 \pm 1.2(13.0-17.5)$ & $15.0 \pm 1.00(13.04-16.96)$ \\
\hline Nabumetone & 12 & $16.5 \pm 2.4(11.9-21.2)$ & $12.0 \pm 3.46(5.21-18.79)$ \\
\hline Celecoxib & 13 & $13.6 \pm 0.8(12.0-15.2)$ & $15.0 \pm 0.87(13.30-16.70)$ \\
\hline
\end{tabular}

Table 3: Wilcoxon (Gehan) test statistics ( $p$-values) for pairwise comparisons of healing times.

\begin{tabular}{|c|c|c|c|}
\hline & Celecoxib & Nabumetone & Naproxen \\
\hline Nabumetone & $0.038(0.847)$ & & \\
\hline Naproxen & $1.03(0.311)$ & $0.094(0.760)$ & \\
\hline Placebo & $5.66(0.017)$ & $1.94(0.164)$ & $3.32(0.069)$ \\
\hline
\end{tabular}

Table 4: Log rank test statistics (p-values) for pairwise comparisons of healing times.

\begin{tabular}{|c|c|c|c|}
\hline & Celecoxib & Nabumetone & Naproxen \\
\hline Nabumetone & $1.17(0.279)$ & & \\
\hline Naproxen & $2.02(0.156)$ & $0.33(0.569)$ & \\
\hline Placebo & $2.77(0.096)$ & $3.86(0.05)$ & $6.58(0.01)$ \\
\hline
\end{tabular}

was skewed to the right, with more people having longer healing times. Since the log-rank test is more sensitive to longer healing times, both naproxen and nabumetone were significant. Results for the Cox, proportional hazards model, were not in agreement with those from the Wilcoxon test but did suggest along with the log-rank test that nabumetone was significant.

\section{Adverse events and symptom complaints}

There were no serious adverse events observed or reported. One subject withdrew from the study after the first endoscopy because of discomfort related to the endoscopy procedure. The following non-serious adverse events or symptoms were documented and all were judged to be either mild or moderate in intensity. Twenty-five subjects reported at least one episode of dyspepsia, (nabumetone $=7$; naproxen $=8$, celecoxib $=6$, placebo $=4$ ). Ten subjects reported at least one episode of headache, (nabumetone $=1$; naproxen $=3$, celecoxib $=4$, placebo=2). Eight subjects reported at least one episode of decreased appetite, (nabumetone $=1$; naproxen $=1$, celecoxib $=5$, placebo $=1$ ). Six subjects reported at least one episode of nausea, (nabumetone $=2$; naproxen $=1$, celecoxib=2, placebo=1). Four subjects reported at least one episode of loose stools or diarrhea, (nabumetone $=1$; naproxen $=0$, celecoxib $=2$, placebo=1). Four subjects reported at least one episode of dizziness or light-headedness, (nabumetone $=1$; naproxen $=3$, celecoxib $=0$, placebo $=0$ ). Four subjects reported at least one episode of taste disturbance, (nabumetone $=2$; naproxen $=0$, celecoxib $=2$, placebo $=0$ ). Two subjects reported at least one episode of vomiting, (nabumetone $=0$; naproxen $=0$, celecoxib $=2$, placebo $=0$ ). Four subjects reported at least one episode of sleep disturbance, (nabumetone $=1$; naproxen $=1$, celecoxib $=0$, placebo $=0$ ). Three subjects reported at least one episode of dryness of the mouth, (nabumetone $=1$; naproxen $=1$, celecoxib $=1$, placebo $=0)$. Three subjects reported at least one episode of pruritus, (nabumetone $=2$; naproxen $=1$, celecoxib $=0$, placebo $=0$ ). Two subjects reported at least one episode of sore throat or pharyngitis, (nabumetone $=1$; naproxen $=1$, celecoxib $=0$, placebo $=0$ ). One subject reported at least one episode of tinnitus, (nabumetone $=0$;
Table 5: Cox proportional hazards regression results. Referent group is based on healing times for the placebo.

\begin{tabular}{|c|c|c|c|c|c|c|c|}
\hline Treatment & $\boldsymbol{\beta}$ & s.e. $(\boldsymbol{\beta})$ & Wald & p-value & RR & \multicolumn{2}{|c|}{$95 \%$ Cl } \\
\hline Celecoxib & -0.424 & 0.389 & 1.18 & 0.276 & 0.654 & 0.305 & 1.403 \\
\hline Nabumetone & -0.95 & 0.447 & 4.52 & 0.034 & 0.387 & 0.161 & 0.929 \\
\hline Naproxen & -0.735 & 0.401 & 3.36 & 0.067 & 0.48 & 0.218 & 1.053 \\
\hline
\end{tabular}

$\beta$ is regression coefficient.

s.e. $(\beta)$ is the standard error of the regression coefficient.

Wald is $\beta$ /s.e. $(\beta)$ and is standard normal distributed.

$\mathrm{RR}$ is relative risk with placebo as referent group, thus RR below one means slower healing.

$95 \% \mathrm{Cl}$ is 95 per cent confidence limit.

naproxen $=1$, celecoxib $=0$, placebo $=0$ ). One subject reported at least one episode of chills, (nabumetone $=0$; naproxen $=1$, celecoxib $=0$, placebo $=0$ ). One subject reported at least one episode of mild dermatitis, (nabumetone $=0$; naproxen $=0$, celecoxib $=1$, placebo $=0$ ). One subject reported at least one episode of increased flatulence, (nabumetone $=0$; naproxen $=1$, celecoxib $=0$, placebo $=0$ ).

\section{Discussion}

The goal of this study was to determine if COX-2 inhibitor therapy would not delay mucosal lesion healing compared to the healing rate with placebo. The secondary goal was to determine if the healing rates with nabumetone were more similar to that of the COX-1 inhibitor, naproxen, or the COX-2 inhibitor, celecoxib. Although the results are not conclusive, they demonstrated a trend favoring celecoxib. The uncertain outcome remains because there are differences between the different statistical test results, and the differences relate to the skewness of each of the healing time distributions. The Wilcoxon test suggests that celecoxib inhibited healing less than nabumetone or naproxen. Surprisingly, the log-rank test suggests that naproxen is much more significant in reducing the healing times, with nabumetone being near the cut-off point for significance $(\mathrm{p}=0.05)$. This analysis is also significant in the Cox regression model. Because of the small sample size, it is difficult to determine if the concomitant H. pylori infection rate, observed among approximately one-third of the subjects in all groups, influenced the outcome of the study.

Overall, it is difficult to conclude with absolute certainty that any of the active drugs delayed healing rates of acute experimental ulcers in humans. Recent studies such as the PRECISION trial report clinical data with prolonged use of either selective COX-2 inhibitors or traditional NSAIDs [15]. They enrolled more than 20,000 patients randomized to receive celecoxib $100-200 \mathrm{mg}$ b.i.d., naproxen $375-500$ $\mathrm{mg}$ b.i.d., or ibuprofen $600-800 \mathrm{mg}$ b.i.d. along with an average of $27 \mathrm{mg}$ of esomeprazole daily (approximately equivalent to $43 \mathrm{mg}$ of omeprazole) and experienced serious gastrointestinal events at a rate of only approximately 5/1000/year [15]. An advantage for celecoxib was evident only in the modified intention-to-treat analysis where the risk, albeit low, was 2 to 3 fold greater with a relatively high dose of PPI with either a traditional NSAIDs than with the selective COX-2 inhibitor plus a PPI. The results with de novo PUBs contrast more markedly with the results of CONCERN study, which studied recurrent gastrointestinal hemorrhage rather than de novo PUBs [22]. They compared esomeprazole $20 \mathrm{mg}$ with $80 \mathrm{mg}$ aspirin daily plus celecoxib $100 \mathrm{mg}$ b.i.d. or naproxen $500 \mathrm{mg}$ b.i.d. for prevention of recurrent ulcer hemorrhage. In that study, rebleeding occurred at a much rate at least 10 times greater (approximately 5.6\%/18 
months with celecoxib vs. $12.3 \%$ with naproxen) than with chronic NSAID in a less at-risk population. Even though they were able to show a difference between selective COX-2 inhibitors and traditional NSAIDs, both resulted in unacceptably high rates of rebleeding. Those data suggest that for chronic ulcers, at least in the immediate post healing stage, neither traditional, nor selective COX-2 inhibitors are safe even when given with relatively high dose PPI (30mg omeprazole equivalent) $[23,24]$

In summary, this study compared traditional NSAIDs and selective COX-2 inhibitors concerning their effect on the healing of acute experimental gastric ulcers in humans. No apparent differences were seen. The differences seen in long-term studies may relate more to differences in ulcerogenesis than in the effects on healing of acute damage $[25,26]$.

\section{Support}

The study was supported in part by a grant from Smithkline Beecham and was performed under IND 58,489. Dr. Graham is currently supported in part by the Office of Research and Development Medical Research Service Department of Veterans Affairs, Public Health Service grant DK56338 which funds the Texas Medical Center Digestive Diseases Center.

\section{Potential Conflicts}

Dr. Graham is a consultant for RedHill Biopharma and Phathom Pharmaceuticals regarding novel $H$. pylori therapies and has received research support for culture of Helicobacter pylori. He is also a consultant for DiaSorin regarding $H$. pylori diagnostics and with Otsuka Japan regarding novel breath tests. He has ongoing collaborative research projects with American Molecular regarding molecular diagnostics for $H$. pylori. He was the PI of an international study of the use of antimycobacterial therapy for Crohn's disease.

\section{References}

1. Graham DY, Chan FK. NSAIDs, risks, and gastroprotective strategies: current status and future. Gastroenterology. 2008; 134: 1240-1246.

2. Laine L. Approaches to nonsteroidal anti-inflammatory drug use in the highrisk patient. Gastroenterology. 2001; 120: 594-606.

3. Jones R. Nonsteroidal anti-inflammatory drug prescribing: past, present, and future. Am J Med. 2001; 110: 4S-7S.

4. Ruszniewski $P$, Soufflet $C$, Barthélémy P. Nonsteroidal anti-inflammatory drug use as a risk factor for gastro-oesophageal reflux disease: an observational study. Aliment Pharmacol Ther. 2008; 28: 1134-1139.

5. Davis A, Robson J. The dangers of NSAIDs: look both ways. The British journal of general practice: the journal of the Royal College of General Practitioners. 2016; 66: 172-173.

6. Venerito M, Wex T, Malfertheiner P. Nonsteroidal Anti-Inflammatory DrugInduced Gastroduodenal Bleeding: Risk Factors and Prevention Strategies. Pharmaceuticals (Basel). 2010; 3: 2225-2237.

7. Sostres C, Gargallo CJ, Arroyo MT, et al. Adverse effects of non-steroidal antiinflammatory drugs (NSAIDs, aspirin and coxibs) on upper gastrointestinal tract. Best. Pract. Res Clin Gastroenterol. 2010; 24: 121-132.
8. Graham DY, Horiuchi A, Kato M. Peptic ulcer disease. In: Yamada T, Alpers DH, Kalloo AN, Kaplowitz N, Owyang C, Powell DW, eds. Yamada's Atlas of Gastroenterology. $4^{\text {th }}$ ed. Hoboken: Wiley-Blackwell. 2008: 237-250.

9. Lanas A, Chan FKL. Peptic ulcer disease. Lancet. 2017; 390: 613-624.

10. Schoen RT, Vender RJ. Mechanisms of nonsteroidal anti-inflammatory druginduced gastric damage. Am J Med. 1989; 86: 449-458.

11. Rainsford KD. Anti-inflammatory drugs in the $21^{\text {st }}$ century. Subcell Biochem. 2007; 42: 3-27.

12. Salzberg DJ, Weir MR. COX-2 inhibitors and cardiovascular risk. Subcell. Biochem. 2007; 42: 159-174.

13. Funk CD, FitzGerald GA. COX-2 inhibitors and cardiovascular risk. J. Cardiovasc. Pharmacol. 2007; 50: 470-479.

14. Nissen SE, Yeomans ND, Solomon DH, et al. Cardiovascular Safety of Celecoxib, Naproxen, or Ibuprofen for Arthritis. N. Engl. J Med. 2016; 375: 2519-2529.

15. Yeomans ND, Graham DY, Husni ME, et al. Randomised clinical trial: gastrointestinal events in arthritis patients treated with celecoxib, ibuprofen or naproxen in the PRECISION trial. Aliment Pharmacol Ther. 2018; 47: 14531463.

16. Graham DY. Nonsteroidal anti-inflammatory drugs, Helicobacter pylori, and ulcers: where we stand. Am. J. Gastroenterol. 1996; 91: 2080-2086.

17. Dahl SL. Nabumetone: a "nonacidic" nonsteroidal antiinflammatory drug. Ann. Pharmacother. 1993; 27: 456-463.

18. Neustadt DH. Double blind evaluation of the long-term effects of etodolac versus ibuprofen in patients with rheumatoid arthritis. J Rheumatol. Suppl. 1997; 47: 17-22.

19. Mizuno H, Sakamoto $\mathrm{C}$, Matsuda $\mathrm{K}$, et al. Induction of cyclooxygenase 2 in gastric mucosal lesions and its inhibition by the specific antagonist delays healing in mice. Gastroenterology. 1997; 112: 387-397.

20. Ma L, del SP, Wallace JL. Divergent effects of new cyclooxygenase inhibitors on gastric ulcer healing: Shifting the angiogenic balance. Proc. Natl. Acad. Sci USA. 2002; 99: 13243-13247.

21. El-Zimaity HM, Ota H, Scott S, et al. A new triple stain for Helicobacter pylor suitable for the autostainer: carbol fuchsin/Alcian blue/hematoxylin-eosin. Arch. Pathol. Lab. Med. 1998; 122: 732-736.

22. Chan FKL, Ching JYL, Tse YK, et al. Gastrointestinal safety of celecoxib versus naproxen in patients with cardiothrombotic diseases and arthritis after upper gastrointestinal bleeding (CONCERN): an industry-independent, double-blind, double-dummy, randomised trial. Lancet. 2017; 389: 23752382.

23. McCarthy DM. Nonprescription Analgesic Anti-inflammatory Drugs: Efficacy and Safety. In: Lanas A, ed. NSAIDs and Aspirin: Recent Advances and Implications for Clinical Management. Cham: Springer International Publishing. 2016: 123-131.

24. Yuan JQ, Tsoi KK, Yang M, et al. Systematic review with network metaanalysis: comparative effectiveness and safety of strategies for preventing NSAID-associated gastrointestinal toxicity. Aliment Pharmacol Ther. 2016; 43: $1262-1275$.

25. Rostom A, Dube C, Wells G, et al. Prevention of NSAID-induced gastroduodenal ulcers. Cochrane Database Syst Rev. 2002: Cd002296.

26. Castellsague J, Riera-Guardia N, Calingaert B, et al. Individual NSAIDs and upper gastrointestinal complications: a systematic review and meta-analysis of observational studies (the SOS project). Drug Saf. 2012; 35: 1127-1146. 